\title{
Comparative Study of Ibuprofen Solubility in Synthetic and Natural Lipid Vehicles
}

\author{
Monzurul Amin Roni and Reza-ul J alil \\ Department of Pharmaceutical Technology, Faculty of Pharmacy, University of Dhaka, \\ Dhaka-1000, Bangladesh
}

Ibuprofen, a non-steroidal anti-inflammatory drug (NSAID), is used in mild to moderate pain and fever. The rapid onset of action of ibuprofen depends on its solubility. Though ibuprofen has high membrane permeability, its solubility in aqueous media is poor $(2.5 \mathrm{mg} / \mathrm{ml}){ }^{1}$ To improve the solubility, several approaches such as solid dispersion ${ }^{2}$, prodrug $^{3}$, inclusion complex ${ }^{4}$, microencapsule ${ }^{5}$ etc. were done for ibuprofen. In the present experiment, solubility of ibuprofen in 2 synthetic and 5 natural lipid vehicles were compared and the effect of nonaqueous solvent (ethanol) on the enhancement of solubility was shown. This solubility analysis is the first step to develop lipid based drug delivery system with any drug. Ibuprofen was obtained from Xamim Co. (China). Propylene glycol monocaprylate (Capmul PG8) was received from Abitec Corp (Janesville, WI), medium chain triglyceride (Neobee M5) was supplied by Stepan Co. (May wood, NJ) and Oleic acid was purchased from Merck (Germany). The other edible vegetable oils were procured locally.

To prepare the standard solution of ibuprofen, 20 $\mathrm{mg}$ of the drug was dissolved in absolute methanol to obtain a concentration of $400 \mu \mathrm{g} / \mathrm{ml}$. The sample was suitably diluted with methanol to obtain concentration in the range of $5-30 \mu \mathrm{g} / \mathrm{ml}$. The $\lambda_{\max }$ of the sample was found at $222 \mathrm{~nm}$ after scanning in UV spectrophotometer (Shimadzu, Japan). The drug

Correspondence to: Reza-ul Jalil

Dhaka Univ. J. Pharm. Sci. 10(1): 65-66, 2011 (June) concentration versus absorption curve was a straight line $\left(\mathrm{R}^{2}=0.99\right)$ having the following formula: $\mathrm{y}=0.0367 \mathrm{x}+0.0253$.

Large excess of ibuprofen ( $3.5 \mathrm{~g}$ ) was taken in centrifuge tubes and $5.0 \mathrm{~g}$ oil phase was added. The mixture was shaken in a vortex mixer for 15 minutes and then stored at room temperature. After 24 hours the undissolved drug sedunented and to obtain clear supernatant the tubes were centrifuged for 5 minutes at $3000 \mathrm{rpm}$. Adequate sample solution was collected and diluted with absolute methanol. Whatman 102 filter paper was used to fiiter the diluted sample which was further suitably diluted with absolute methanol to determine the concentration of drug in saturated solution. The samples were analyzed with UV spectrophotometer (Shimadzu, Japan) at $222 \mathrm{~nm}$ against methanol as blank.

To determine the effect of ethanol on the solubility of ibuprofen-oil mixture, initially $0.5 \mathrm{~g}$ of absolute ethanol ( $10 \%$ of oil phase) was added in the saturated solution. According to the procedure mentioned above the concentration of drug in oil was determined by UV analysis. The analysis was repeated for increased amount of ethanol (20\% and $30 \%$ of oil phase). In the study, ibuprofen showed better solubility in oleic acid (Table 1) than other natural oils. The higher solubility of ibuprofen in oleic acid was not reflected in case of olive oil (Table 1), though it contains $75 \%$ of oleic acid, ${ }^{6}$ because the oil also contains glycerol.

Propylene glycol monocaprylate has shown highest solubility (Table 1) among the synthetic lipid 
vehicles. It is primarily used as a carrier in soft and hard gelatin capsules and a penetration enhancer in transdermal drug delivery systems. The solubility of drug in oleic acid and propylene glycol monocaprylate was similar.

Table 1. Solubility of ibuprofen in different lipid vehicles $(n=2$, mean standard error)

\begin{tabular}{lc}
\hline Solvent & $\begin{array}{c}\text { Solubility at } 2 \mathrm{~S}^{\circ} \mathrm{C} \\
(\mathrm{mg} / \mathrm{ml}), 0=2, \pm \text { S.E. }\end{array}$ \\
\hline $\begin{array}{l}\text { Medium chain triglyceride } \\
\text { (Neobee M5) }\end{array}$ & $149.00 \pm 2.11$ \\
$\begin{array}{l}\text { Propylene glycol monocaprylate } \\
\text { (Capmul PG8) }\end{array}$ & $326.40 \pm 0.15$ \\
Arachis oil & $113.40 \pm 2.03$ \\
Castor oil & $223.70 \pm 1.42$ \\
Oleic acid & $311.40 \pm 0.01$ \\
Olive oil & $153.30 \pm 0.57$ \\
Soybean oil & $96.30 \pm 1.55$ \\
\hline
\end{tabular}

Ethanol was added to improve solubility of ibuprofen in the hydrophobic media. The solubility increased in oleic acid initially upon the addition of $10 \%$ (w/w of oil) ethanol but did not improved significantly when more of the ethanol was added (Figure 1). Similar phenomena was observed for propylene glycol monocaprylate, where addition of ethanol did not improved drug solubility.

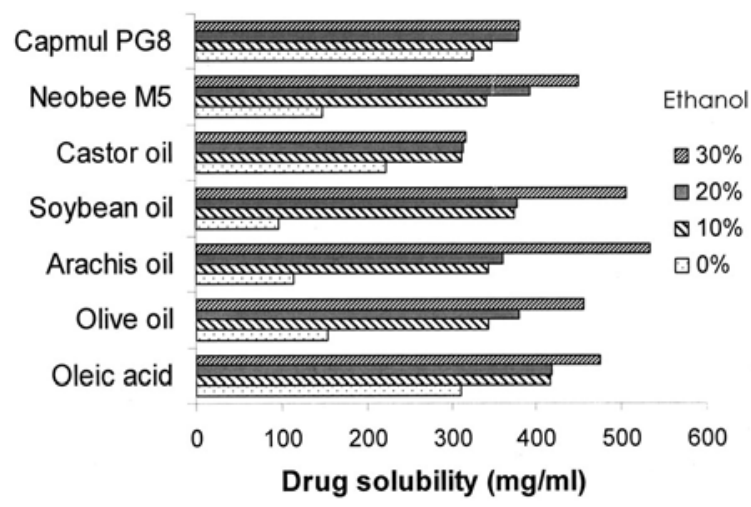

Figure 1. Effect of ethanol concentration on the solubility of ibuprofen in lipid vehicles.

On the other hand, soybean oil performed poorly as a solvent but when ethanol was added, the solubility increased rapidly. Similar trend was seen for arachis oil, olive oil and medium chain triglyceride (Figure 1). The sudden increase in solubility is due to the higher solubility of drug in ethanol.

To conclude, among the lipidic vehicles tested, the performance of synthetic and natural solvents did not differ widely. Oleic acid and propylene glycol monocaprylate showed highest solubility of ibuprofen. Their solubility can be improved up to some extent with the help of ethanol. The solubility of ibuprofen can be increased significantly in case of soybean oil, medium chain triglyceride, olive oil and arachis oil with the help of ethanol. As ibuprofen is a high dose drug (1.2-1.6 $\mathrm{g}$ daily $)^{7}$ and ethanol is restricted in oral formulation, oleic acid and propylene glycol monocaprylate are more suitable for the formulation of soft gelatin capsule of ibuprofen.

\section{REFERENCES}

1. Ahn, Y.S., Song, I.H., Kang, B.K., Kim, M.S., Cho, S.H., Rhee, IM., Lee, H.B. and Khang, G. 2004. Preparation and characterization of liquefied Ibuprofen using selfmicroemulsion drug delivery system (SMEDDS). J. Kor. Pharrn. Sci. 34, 35-42.

2. Newa, M., Bhandari, K.H., Kim, J.O., I.M, J.S., Kim, J.A., Yoo, B.K., Woo, J.S., Choi, H.G. and Yong, C.S. 2008. Enhancement of solubility, dissolution and bioavailability of ibuprofen in solid dispersion systems. Chem. Pharm. Bull. 56, 569-574.

3. Murtha, J.L. and Ando, H. Y1994. Synthesis of the cholesteryl ester prodrugs cholesteryl ibuprofen and cholesteryl flufenamate and their formulation into phospholipid micro-emulsion. J. Pharm. Sci. 83, 1222-1228.

4. Ghorab, M.K. and Adeyeye M.C. 2001. Enhancement of ibuprofen dissolution via wet granulation with betacyclodextrin. Pham. Dev. Tech. 6, 305-314.

5. Adeyeye, C.M. and Price, I.C. 1994. Development and evaluation of sustained-release ibuprofen-wax microspheres. II. In vitro dissolution studies. Pharm. Res. 1, 575-579.

6. Tyler, V.E., Brady, L.R. and Robbers, J.E. 1981. Pharmacognosy. Lea \& Febiger, Philadelphia, $9^{\text {th }}$ ed. p. 89.

7. Bangladesh National Formulary 2006. Directorate of Drug Administration, p. 302. 